\title{
Survival trends from the Prader-Willi Syndrome Association (USA) 40-year mortality survey
}

\author{
Ann M. Manzardo, PhD ${ }^{1}$, James Loker, MD², Janalee Heinemann, MSW ${ }^{3}$, Carolyn Loker ${ }^{3}$ and \\ Merlin G. Butler, MD, PhD ${ }^{1}$
}

Purpose: Prader-Willi syndrome (PWS) is a complex genetic disorder characterized by hyperphagia and morbid obesity with increased cardiopulmonary and hyperphagia-related mortality. Survival trends in PWS were evaluated to assess the impact of modern interventions on mortality risk.

Methods: The Prader-Willi Syndrome Association (USA) 40-year mortality syndrome-specific database of 486 death reports was utilized to examine survival trends in PWS and cohort effects for recent deaths (years 2000-2015, N=331) relative to deaths prior to $2000(N=94)$. Cox proportional hazards regression modeling was applied to generate log rank statistics and Kaplan-Meier curves examining sex, cause of death, and cohort.

Results: Risk for all-cause mortality in PWS was 1.5 (95\% confidence interval $(\mathrm{CI})=1.2-1.9)$ times higher for the Early than the Recent era cohort reflected in female cardiac failure (hazard ratio $(\mathrm{HR})=1.8 ; 95 \% \mathrm{CI}=1.3-2.6)$, pulmonary embolism $(\mathrm{HR}=$ $6.1 ; 95 \% \mathrm{CI}=1.7-22)$, and gastrointestinal-related $(\mathrm{HR}=3.2 ; 95 \%$ $\mathrm{CI}=1.1-7.4)$ causes. Accidental deaths in males increased in the Recent era cohort $(\mathrm{HR}=5.7 ; 95 \% \mathrm{CI}=1.2-27.1)$, possibly due to enhanced weight management and mobility. Risk of death from respiratory failure was unchanged.

Conclusion: We report measurable increases in survival effecting cardiovascular and gastrointestinal-related causes in PWS most likely attributable to earlier diagnosis and proactive interventions to prevent morbid obesity. More research is needed to address underlying vulnerability to respiratory failure, an unchanged mortality risk in PWS.

Genet Med advance online publication 6 July 2017

Key Words: mortality; obesity; Prader-Willi syndrome; thromboembolism

\section{INTRODUCTION}

Prader-Willi syndrome (PWS) is a complex neurodevelopmental genetic disorder with multiple cognitive, behavioral, and endocrine abnormalities caused by errors in genomic imprinting generally due to lack of paternally expressed genes from the $15 \mathrm{q} 11-\mathrm{q} 13$ region. ${ }^{1-3}$ First identified in 1956, PWS is considered the most common known genetic cause of lifethreatening obesity in humans with a prevalence of 1 in 10,000 to 30,000 live births. ${ }^{1-5}$ A shortened life expectancy is found in relationship to the level of intellectual disability primarily from complications of hyperphagia and obesityrelated comorbidities. ${ }^{5,6}$

PWS is characterized by severe central hypotonia, a poor suck and feeding difficulties, and hypogonadism and hypogenitalism observed in both males and females beginning in infancy. ${ }^{7}$ Growth and other hormone deficiencies lead to growth failure, short stature, small hands and feet, infertility, and endocrine disturbances. ${ }^{1,2,8}$ Hypothalamic dysfunction produces temperature instability, high pain threshold, sleep disturbances, and multiple endocrine problems. ${ }^{8-10}$ Infants with PWS are at risk for respiratory problems due to a narrow upper airway, possible aspiration, central hypotonia, hypoventilation, a dry mouth with sticky saliva, and swallowing difficulties with gastroenteritis, which complicate medical care and response to treatment, requiring close monitoring throughout life. . $^{1-3,7,10-12}$

Hyperphagia or the unrelenting pathologic urge to consume food with unremitting hunger in PWS typically develops in childhood, leading to excessive food-seeking behavior and life-threatening obesity, if not controlled. ${ }^{1-3,7,13}$ The risk for obesity is exacerbated by a low basal metabolic rate that is approximately $60 \%$ of normal, decreased lean muscle mass, and increased fat mass related to endocrine abnormalities. ${ }^{1,2,14}$ Intractable hyperphagia in PWS continues throughout adulthood with limited options for treatment and significantly diminishes the quality of life for those affected and for family members. Developmental delays and mild intellectual disability are noted with behavioral problems recognized in childhood including tantrums, stubbornness, obsessive compulsions, and self-injury (skin picking) that continue into adolescence and adulthood. ${ }^{1-4}$ Morbid obesity is a significant contributor to mortality in PWS through cardiorespiratory failure, ${ }^{10}$ pulmonary thromboembolism, and associated diabetes with multiorgan failure. Hyperphagia-related behaviors also increase the risk for gastrointestinal perforation and necrosis, aspiration, choking, and swallowing difficulties 
due to a combination of factors from central hypotonia, reduced gastrointestinal (GI) motility, and rapid food consumption. ${ }^{11-13}$ Additionally, excoriation can create severe wounds and precipitate life-threatening infections.

Modern genomic technology has facilitated the detailed genetic characterization of PWS and its subtypes and enabled diagnosis of PWS in infancy. ${ }^{14-17}$ Earlier diagnosis has increased the opportunity for treatment and intervention to control access to food, reduce risks, and prevent the onset of obesity. Growth hormone was first used to treat short stature in PWS in the 1980s and had limited use in patients with PWS until approved by the US Food and Drug Administration in 2000 to increase stature and leads to lean muscle mass and reduce risk of obesity. ${ }^{18-20}$ Growth hormone is now widely utilized to treat PWS in the United States beginning at birth through adulthood. ${ }^{18-20}$ We anticipate that increased diagnosis and earlier interventions associated with this modern era have improved the long-term outcomes for individuals with PWS; however, recent longitudinal analyses of mortality rates in PWS still indicate a significantly shortened lifespan. ${ }^{19-21}$ The present study examines the impact of cohort era prior to and after 2000 on causes of death and mortality in PWS. We report survival trends and analysis of the largest collection of causes of death in PWS utilizing the Prader-Willi Syndrome Association (PWSA (USA)) syndrome-specific mortality survey database spanning the past 40 years.

\section{MATERIALS AND METHODS}

The Prader-Willi Syndrome Association (PWSA (USA)) is a nonprofit parent-support organization founded by PWS families to supply information and assist or support families and others caring for those affected with this disorder. Data on causes of death in PWS have been collected and recorded by the PWSA (USA) since 2001 through a supportive bereavement program for PWS families. ${ }^{22}$ The data includes descriptive information collected through a brief survey administered by the bereavement coordinator. An additional detailed questionnaire with a release of medical records including autopsy reports was disseminated to families known to have experienced the death of a relative with PWS in 2005. Genetic confirmation of PWS diagnosis was obtained when available; however, the PWS deletion seen in about $70 \%$ of those with PWS was not discovered until 1980 and genetic confirmation using DNA methylation testing, which is $99 \%$ accurate, was not available until $1992 .{ }^{15}$ Thus, deaths occurring prior to 1992 and many older adults may not have been genetically confirmed. The collected data were assembled into a database, reviewed by PWS experts, and cleaned to correct errors. The reported causes of and contributors to each death and autopsy data were evaluated clinically by a licensed cardiologist, parent, and expert in the care of PWS who assessed the primary cause of death for each individual. The defined causes of death were then classified into 13 major categories as described in 2017 by Butler et al. ${ }^{22}$

\section{Statistical analysis}

The data set included 486 ( $N=263$ males, $N=217$ females, $N=6$ sex unknown) individuals with PWS. ${ }^{22}$ A precise cause of death was available for $311(N=173$ males, $N=137$ females, $N=1$ sex unknown) and a precise age of death was known for $425(N=224$ males, $N=199$ females, $N=2$ sex unknown). Both the age and cause of death were available for 302 ( $N=166$ males, $N=135$ females, $N=2$ sex unknown) individuals. ${ }^{22}$ Kaplan-Meier plots and log-rank statistics were examined and Cox proportional hazards regression was applied to assess the risk of all-cause mortality as a function of each reason of cause, gender, and their interactions. Timeto-event outcomes were analyzed with hazard ratios as estimates of cumulative hazard or risk of death at time $t$ as a measure of the relative death rate. Analyses were carried out using the total data set with the data available for individual variables under study to maximize statistical power.

The impact of historical time frame on mortality risk was evaluated by dividing the sample into two categories: Recent deaths-occurring from 2000 to 2015 with $N=331(N=171$ male, $N=159$ females, $N=1$ sex unknown) cases having a known age of death. Of these, $N=254$ ( $N=140$ males, $N=113$ females, $N=1$ sex unknown) individuals had both a known age and cause of death; and Early deaths-occurring prior to the year 2000 beginning in 1975 with $N=94(N=53$ male, $N=40$ females, $N=1$ sex unknown) cases having a known age of death. Of these, $N=57(N=33$ males, $N=24$ females) individuals had both a known age and cause of death. Chi-squared tests, Kaplan-Meier plots and log-rank statistics were examined and Cox proportional hazards regression was applied to assess the risk of all-cause mortality as a function of cohort era, gender, and their interactions. All statistical comparisons were performed using SAS Statistical Software version 9.4 (Cary, NC).

\section{RESULTS}

Cox proportional hazards regression analysis of individual causes of death relative to all-cause mortality for $N=224$ males and $N=199$ females with PWS found the risk of death due to respiratory failure to be significantly increased over all causes (log-rank $\chi^{2}=8.2, \mathrm{df}=1 ; p<0.004$; hazard ratio $(\mathrm{HR})=1.4 ; 95 \% \mathrm{CI}=1.1-1.8)$, which was attributable to a sex-specific increase in risk of respiratory failure in females $(\mathrm{HR}=2.0,95 \% \mathrm{CI}=1.4-2.8$ females; $\mathrm{HR}=1.1,95 \% \mathrm{CI}=$ 0.76-1.5 males; see Figure 1a, Table 1) A sex-specific increase in risk of accidental death was also observed in males $(\mathrm{HR}=2.2,95 \% \mathrm{CI}=1.2-4.0$ males; $\mathrm{HR}=0.8,95 \% \mathrm{CI}=0.34-$ 1.8 females; see Figure 1b, Table 1). No other differences were observed for individual causes of death relative to all-cause rates or by gender.

\section{Time era cohort effects in PWS}

Examination of era cohort effects on survival outcomes identified a significantly greater risk of death due to all causes for the Early era cohort (deaths occurring prior to 2000) relative to the Recent era PWS cohort (years 2000-2015) with 


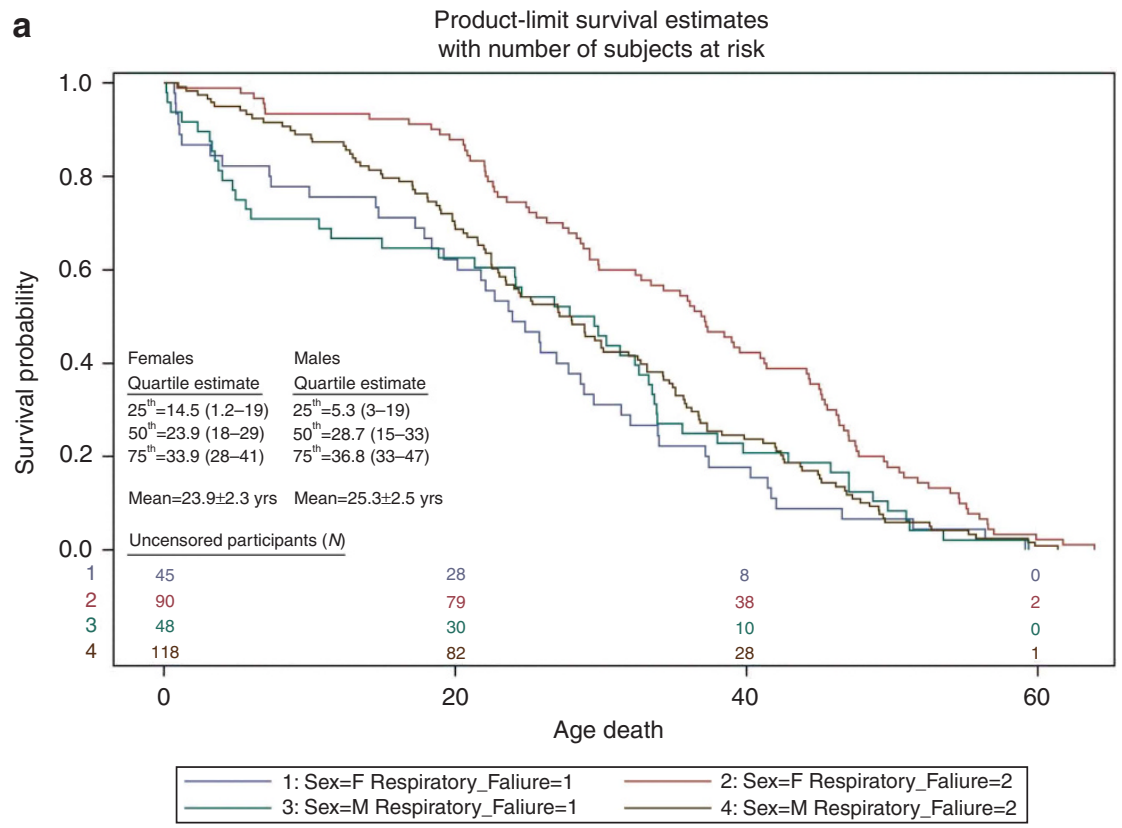

Accidental deaths

b

Product-limit survival estimates with number of subjects at risk

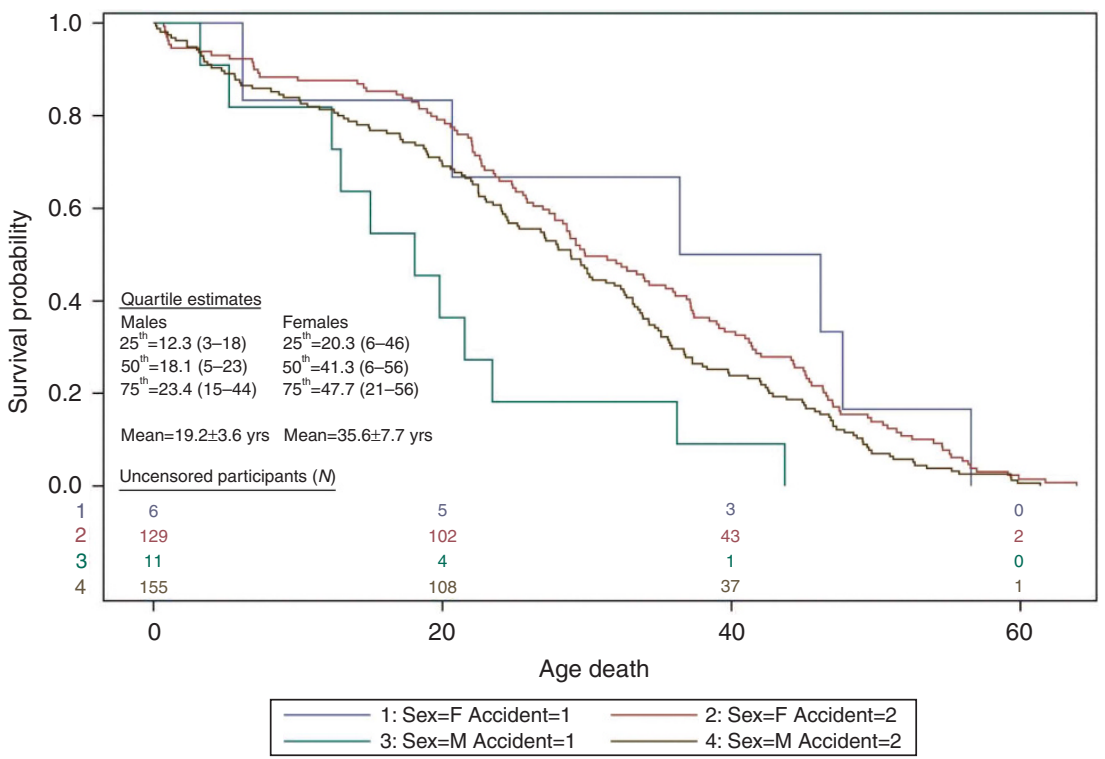

Figure 1 Kaplan-Meier plots of survival probability. (a) Plot of deaths due to respiratory failure (Respiratory Failure (1)) relative to all other causes (Respiratory Failure (2)) in Prader-Willi syndrome are shown for females (F) and males (M). The number of uncensored participants are listed by group at the bottom of each figure. Quartile estimates for age of death and mean age of mortality due to respiratory failure are indicated. (b) Plot of survival probability for accidental deaths (Accident (1)) relative to all other causes (Accident (2)) in Prader-Willi syndrome for females (F) and males (M). The number of uncensored male and female participants are listed at the bottom of each figure. Quartile estimates for age of death and mean age of mortality are indicated for males and females.

an overall hazard of Early death that was 1.5 times greater than that for Recent death $(\mathrm{HR}=1.5,95 \% \mathrm{CI}=1.2-1.9$; logrank $\left.\chi^{2}=13.2, \mathrm{df}=1, p<0.0003\right)$. This corresponded with a gender-selective increase in risk of female deaths in the Early cohort $(\mathrm{HR}=1.8$ Early death; $95 \% \mathrm{CI}=1.3-2.6)$ not found for males ( $\mathrm{HR}=1.3$ Early death; 95\% CI $=0.96-1.8$; see Figure 2; Table 1). This association paralleled an increased risk for cardiac deaths among females in the Early era cohort $(\mathrm{HR}=1.8$ Early death; $95 \% \mathrm{CI}=1.3-2.6$; see Table 1$)$. The overall frequency of cardiac deaths was significantly higher in the Early era cohort (15 of $57(26 \%)$ ) than the Recent era cohort (36 of $254(14 \%)) \quad(\mathrm{OR}=2.2 ; 95 \% \mathrm{CI}=1.1-4.3$; $\left.\chi^{2}=5.0, p<0.03\right)$ supporting a trend toward reduced overall cardiac death in the PWS population. Evaluation of cohort 
Table 1 Cox proportional hazard regression modeling Respiratory failure vs. all-cause mortality

\begin{tabular}{lccl}
\hline Global test & Chi-square & $\begin{array}{c}\text { Differential } \\
\text { function }\end{array}$ & $\boldsymbol{P}$ value \\
\hline Likelihood ratio & 18.1 & 3 & 0.0004 \\
Type 3 test & & & \\
Respiratory failure & 0.16 & 1 & 0.69 \\
Female sex & 9.7 & 1 & 0.002 \\
Female sex $\times$ respiratory failure & 5.9 & 1 & 0.01
\end{tabular}

\begin{tabular}{lccc}
\hline \multicolumn{4}{l}{ Accidental deaths vs. all-cause mortality } \\
\hline Global test & Chi-square & DF & P value \\
\hline Likelihood ratio & 10.9 & 3 & 0.01 \\
Type 3 test & & & \\
Accidental deaths & 6.1 & 1 & 0.01 \\
Sex & 3.6 & 1 & 0.06 \\
Male sex $\times$ accidental death & 3.8 & 1 & 0.05 \\
\hline
\end{tabular}

\begin{tabular}{|c|c|c|c|}
\hline \multicolumn{4}{|c|}{ Risk of death for early vs. recent cohorts } \\
\hline Global test & Chi-square & DF & $P$ value \\
\hline Likelihood ratio & 17.9 & 3 & 0.0005 \\
\hline \multicolumn{4}{|l|}{ Type 3 test } \\
\hline Early vs. recent deaths & 2.9 & 1 & 0.08 \\
\hline Female sex & 5.6 & 1 & 0.02 \\
\hline Female sex $\times$ early deaths & 1.9 & 1 & 0.1 \\
\hline
\end{tabular}

Risk of death from cardiac failure for early vs. recent cohorts

\begin{tabular}{lccc}
\hline Global test & Chi-square & DF & P value \\
\hline Likelihood ratio & 17.9 & 3 & 0.0005 \\
Type 3 test & & & \\
Early vs. recent deaths & 2.9 & 1 & 0.08 \\
Female sex & 5.6 & 1 & 0.02 \\
Female sex $\times$ early deaths & 1.9 & 1 & 0.2
\end{tabular}

Risk of death from accidents for recent vs. early cohorts

\begin{tabular}{lccc}
\hline Global test & Chi-square & DF & $\boldsymbol{P}$ value \\
\hline Likelihood ratio & 23.8 & 3 & 0.0001 \\
Type 3 test & & & \\
Early vs. recent deaths & 15.2 & 1 & 0.0001 \\
Accidental death & 5.7 & 1 & 0.017 \\
Accidents $\times$ early deaths & 14.3 & 1 & 0.0002 \\
\hline
\end{tabular}

$\mathrm{DF}$, degrees of freedom.

effects for individual causes of death are limited by complexity and small sample size particularly in the Early era cohort. No other differences were noted in the frequency of any individual cause of death or sex by cohort.

We identified an unexpected increase in the risk of early mortality due to accidental death among the Recent era cohort $(\mathrm{HR}=5.2 ; 95 \% \mathrm{CI}=1.7-16.0$ for Recent era relative to Early era cohorts; see Table 1; Figure 3a) driven by an effect in males $(\mathrm{HR}=5.7$ Recent death; $95 \% \mathrm{CI}=1.2-27.1)$. This unexpected increase in risk for accidental deaths among males may have offset decreases in mortality risk associated with improvements in other hemodynamic factors (e.g., cardiac failure, thromboembolism). The specific risk of accidental death among females could not be analyzed due to the small number of deaths $(N=6)$. Disease-specific examination without consideration of gender or competing risk from other causes of death found the risk of death due to pulmonary embolism $(\mathrm{HR}=6.1$ Early death; $95 \% \mathrm{CI}=1.7$ 22; $\left.\chi^{2}=7.5 ; p<0.006\right)$ and GI-related complications (HR $=$ 3.2 Early death; $\left.95 \% \mathrm{CI}=1.1-7.4 ; \chi^{2}=4.5 ; p<0.03\right)$ to be significantly higher in the early era cohort than the Recent era cohort but the risk of death due to respiratory failure and choking were not different. Controlled regression modeling with consideration of competing effects of other causes of death did not achieve significance (see Figure $3 \mathbf{b}$ ).

\section{DISCUSSION}

This detailed investigation of survival trends and risk factors extends our previous characterization of mortality age, causes, and related factors in PWS and identifies gender-specific influences and causal risk factors leading to early mortality observed in PWS. ${ }^{22}$ Respiratory failure has emerged as a leading contributor to mortality in PWS manifesting very early in life for both males (mean age $=25.3 \pm 2 \mathrm{yr}$ ) and females (mean age $=23.9 \pm 2 \mathrm{yr}$ ). Reported cases of respiratory failure were not correlated with obesity, typically reported as unexpected (82\%) contributors to death by family members, and often associated with prior complaints of minor illness, respiratory distress, or associated emergency room visits. More research is needed to characterize the underlying pathology for these sudden deaths. As previously shown, males are at increased risk for early mortality due to behavioral or activity-oriented causes (e.g., accidents, choking) which may reflect gender differences in activity level, behavioral activation, or manifestation of hyperphagia. ${ }^{22}$

Previous regression modeling in this sample identified quartile point estimates for all-cause mortality with 50\% mortality at 29 years of age (95\% CI (27-32 yr)) and 75\% mortality at 42 years of age $(95 \% \text { CI }(39-44 \text { yr }))^{22}$ Examination of Recent versus Early era mortality trends paints a more optimistic future for PWS, revealing clear gender- and disease-specific increases in long-term survival trends since 2000. Major contributors to early mortality in PWS-cardiac failure and the recently identified risk of thromboembolism-were significantly delayed and/or reduced in recent years along with gastrointestinal complications. ${ }^{6,23-29}$ Increased awareness, early diagnosis, dietary intervention with restricted caloric intake, and significant controls on access to food (locking cabinets, refrigerators) with established exercise programs for each person with PWS have led to successful weight loss and body mass index reduction. Earlier diagnosis is expected to correlate with increased physical activity and strength as well as exposure to growth hormone, which was approved for use in PWS in 2000. The use of growth and other hormone replacements have helped to normalize body composition and stature in PWS with a positive impact on the control of obesity. ${ }^{18-21}$ Our data provides empirical evidence that active management of obesity and hyperphagia in PWS does prolong life. 
Mortality risk in the Recent era timeframe appears to reflect a shift away from obesity-related causes to other behavioral and/or biological factors not previously recognized or as closely monitored, such as blood clotting. Furthermore, morbid obesity, more frequently observed in the Early era cohort, often led to physical immobility, which precipitated cardiopulmonary failure. Recent era changes in medical management including general medical care for obesity and cardiovascular disease, and reduced rates of morbid obesity including tight caloric restrictions in combination with an improved ability to be active, appear to have increased the likelihood of fatal accidents in males with PWS. Decreased weight coupled with the effects of growth hormone, increased muscle mass, activity level, and social engagement provides more avenues for behavioral, hyperphagia-related problems and other accidents. Males with PWS appear to be more likely to engage in risky behaviors. It is not clear whether the tight caloric restrictions employed in the management of PWS have impacted food drive or seeking behaviors to impact risk.

The results of our study support the need for increased awareness, and close monitoring and supervision during meals, outings, and near roadways to reduce the risk of traffic collision from food seeking (e.g., darting into the road to reach a restaurant). Strict oversight of food access and quantity are critical to prevent excessive unsupervised food consumption, choking, and gastric rupture, which can occur from eating quickly. Chewing and swallowing may be compromised in PWS by decreased salivary secretions, dental caries, a poor muscle tone, and reduced sensation with lack of vomiting. ${ }^{1-5}$ Implementation of preventive measures, specialized training, and better awareness of families and group home care providers of these risks are recommended for all individuals with PWS including training for use of the Heimlich maneuver, supervised meals, and food security along with food preparation and diet modification. The

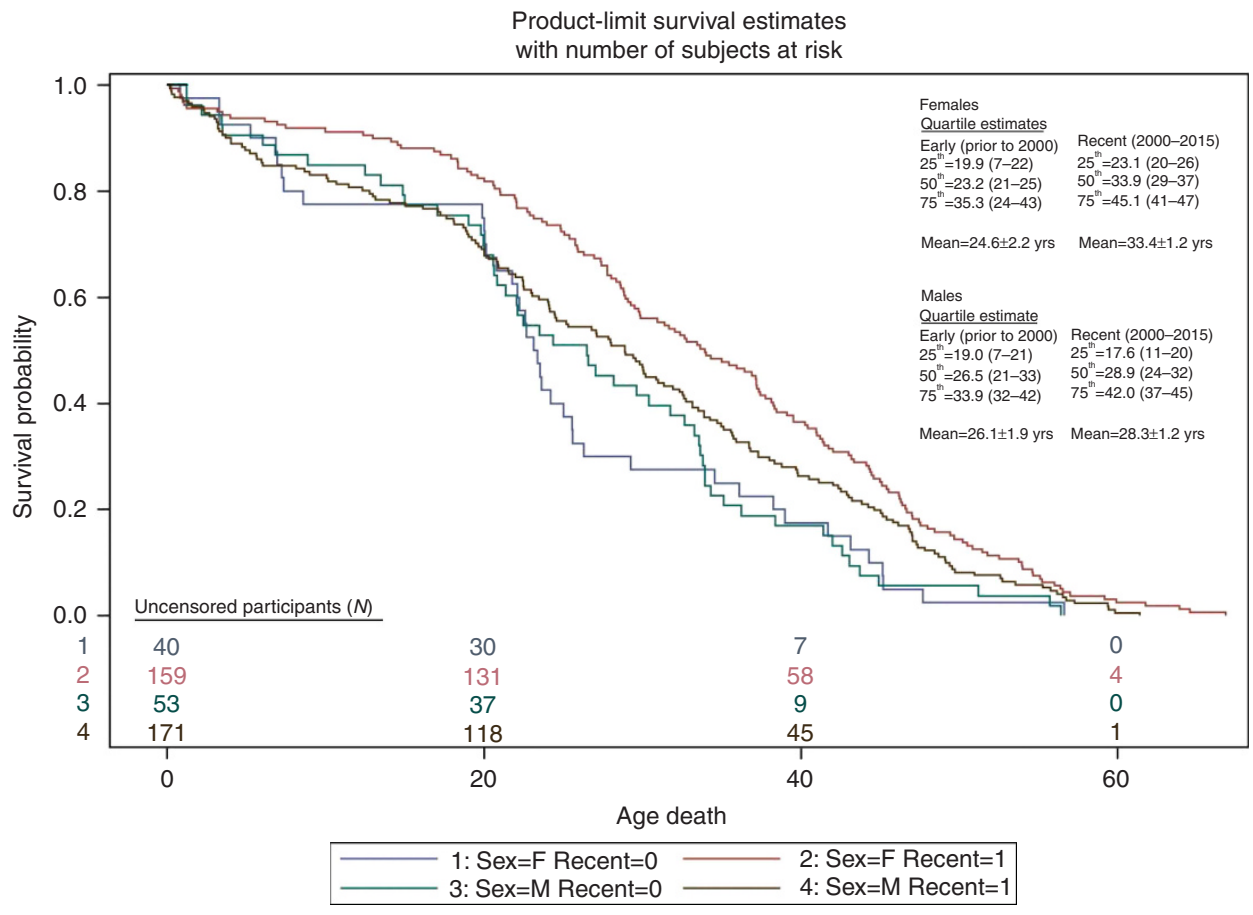

Figure 2 Cohort effects on all-cause mortality in Prader-Willi syndrome. Kaplan-Meier plot of survival probability for deaths due to all causes for Early era (Recent (0)) and Recent era (Recent (1)) cohorts with Prader-Willi syndrome are shown for females (F) and males (M). The numbers of uncensored Recent and Early era participants are listed at the bottom of the figure. Quartile estimates for age of death and mean age of mortality are indicated for Recent and Early era groups.

Figure 3 Kaplan-Meier plots of survival probability. (a) Plot of deaths due to Accidents (Accident (1)) relative to all other causes (Accident (2)) in Prader-Willi syndrome for Recent era (Recent (1)) versus Early era (Recent (0)) cohorts are shown. The numbers of uncensored Recent and Early era participants are listed at the bottom of each figure. Quartile estimates for age of death and mean age of mortality are indicated for Recent and Early era groups with pulmonary embolism. (b) Plot of survival probability for deaths due to pulmonary embolism (Pulmonary_Embolism (1)) (top) and gastrointestinal (GI) problems (Gastrointestinal (1)) (bottom) relative to all other causes (Pulmonary_Embolism (2); Gastrointestinal (2)) in Prader-Willi syndrome for Recent era (Recent (1)) versus Early era (Recent (0)) are shown. The numbers of uncensored Recent and Early era participants are listed at the bottom of each figure. Quartile estimates for age of death and mean age of mortality are indicated for Recent and Early era groups for gastrointestinal problems or accidents respectively. 


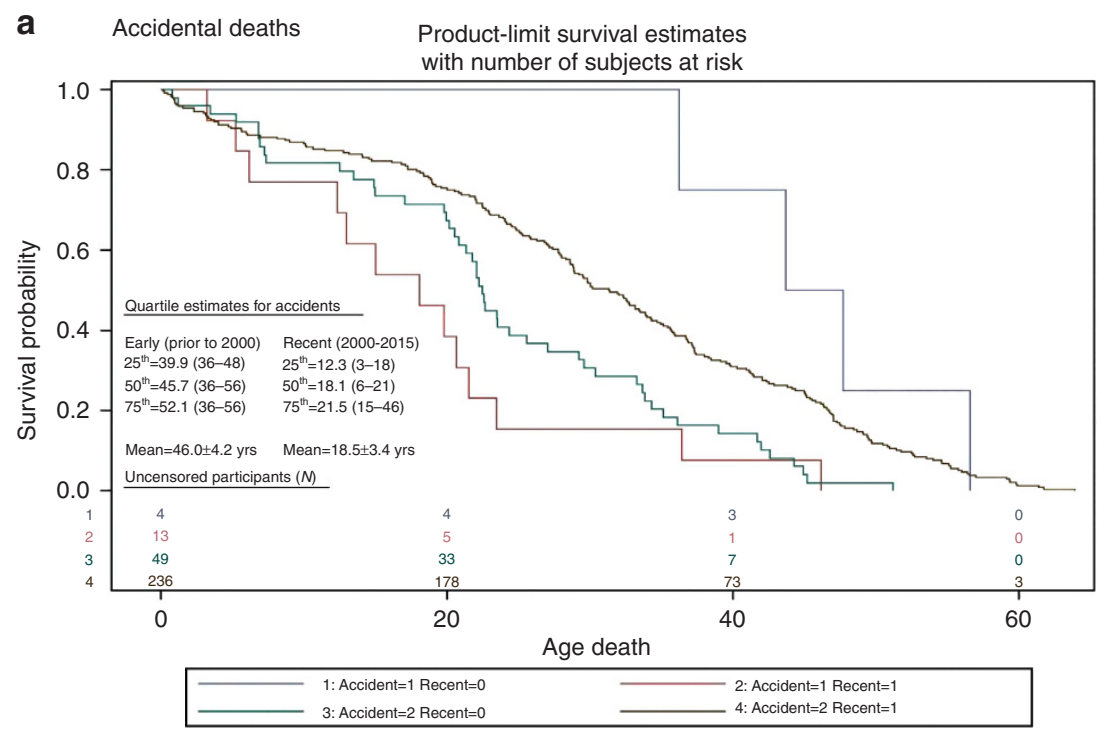

b Pulmonary Embolism $\quad \begin{aligned} & \text { Product-limit survival estimates } \\ & \text { with number of subjects at risk }\end{aligned}$

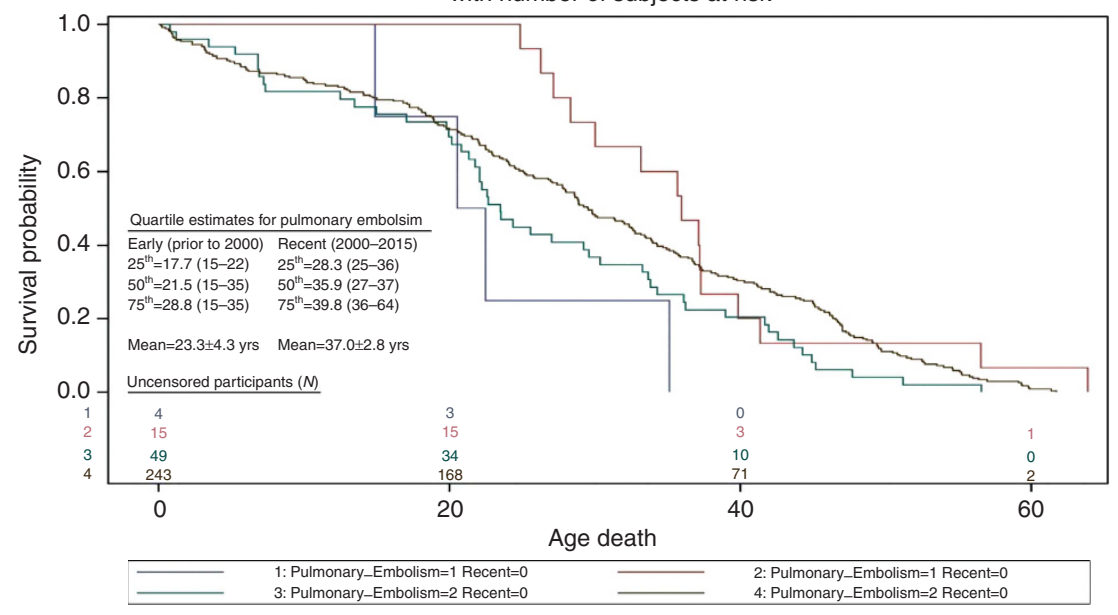

Gastrointestinal Problems

Product-limit survival estimates with number of subjects at risk

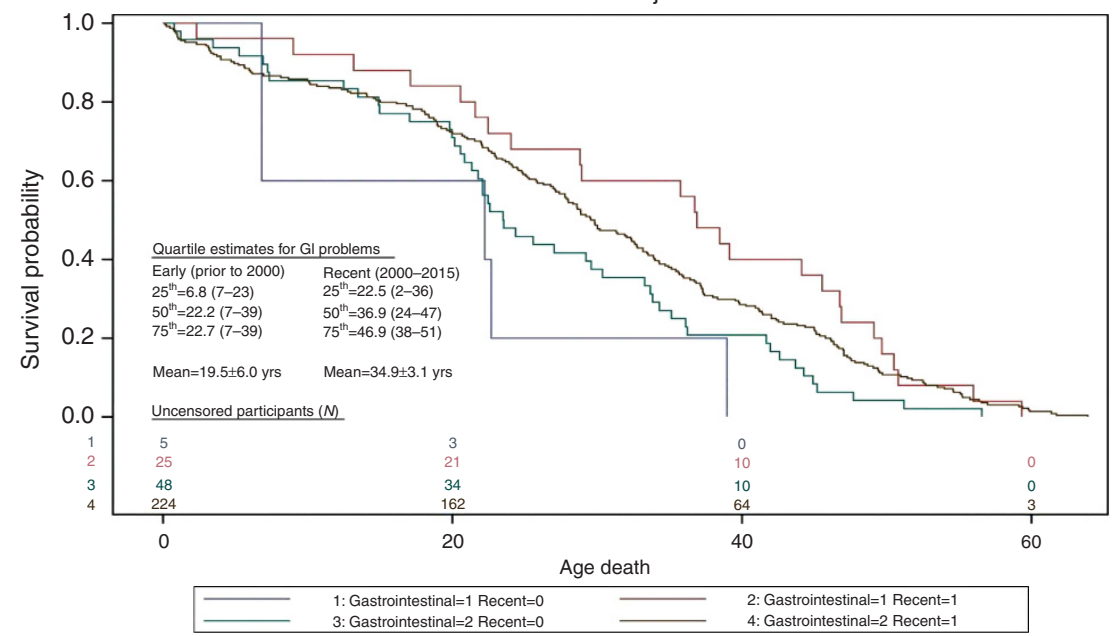


necessity for lifelong weight control and vigilant food restrictions to prevent excessive eating, overweight, and lifethreatening obesity in PWS is now well understood among most families and medical experts. Further, unrestricted access to food provides no relief from the insatiable food cravings experienced in PWS and only serves to exacerbate health risks and physical disability, ultimately leading to the loss of the fundamental freedom afforded by the good health, mobility, and longevity that is associated with leanness. The present study findings support increases in longevity in PWS with appropriate management and care.

\section{Conclusions}

In summary, the present survival analysis extension study represents the largest and most extensive examination of survival trends in PWS to date and the results support current understanding of disease pathology and mortality in PWS. Current survival estimates for PWS are significantly increased from those prior to 2000 particularly for cardiac deaths among females as well as thrombotic and GI-related mortality. Males may be more likely to engage in aggressive or risky food-seeking behaviors than females leading to greater risk of accidental deaths such as traffic collision, choking, or GI perforation. Our analyses and interpretation of data trends are limited by the reliability of death reporting based upon the availability and knowledge of family members and access to confirmed genetic status and autopsy reports. Infants and children with PWS from the Early era cohort may have escaped diagnosis prior to death and older individuals may have been underreported or misdiagnosed. Nevertheless, the results provide useful insight into risk factors, mortality trajectory over time, and areas of need. Family members, care providers, and health-care professionals involved with the immediate and long-term care of individuals with PWS should be made aware of these risk factors and causation of death to improve the longevity and quality of life for those with PWS (at all ages) and family members.

\section{ACKNOWLEDGMENTS}

We acknowledge the support of National Institute of Child Health and Human Development grant number HD02528. Partial funding was also received in an unrestricted grant from Zafgen. We also acknowledge the support of the Prader-Willi Syndrome Association (USA) and families.

\section{DISCLOSURE}

The authors declare no conflicts of interest.

\section{REFERENCES}

1. Butler MG. Prader-Willi syndrome: current understanding of cause and diagnosis. Am J Med Genet. 1990;35:319-332.

2. Butler MG, Lee PDK, Whitman BY (eds). Management of Prader-Willi Syndrome, 3rd edn. Springer: New York, 2006.
3. Cassidy SB, Schwartz S, Miller JL, Driscoll DJ. Prader-Willi syndrome. Genet Med 2012;14:10-26.

4. Hoybye C (ed). Prader-Willi syndrome. In: Congenital Disorders: Laboratory and Clinical Research. Nova Science: New York, 2013.

5. Butler JV, Whittington JE, Holland AJ, Boer H, Clarke D, Webb T. Prevalence of, and risk factors for, physical ill-health in people with Prader-Willi syndrome: a population-based study. Dev Med Child Neurol. 2002:44:248-255.

6. Whittington JE, Holland AJ, Webb T, Butler J, Clarke D, Boer H. Population prevalence and estimated birth incidence and mortality rate for people with Prader-Willi syndrome in one UK health region. J Med Genet. 2001;38:792-798.

7. Angulo MA, Butler MG, Cataletto ME. Prader-Willi syndrome: a review of clinical, genetic, and endocrine findings. J Endocrinol Invest. 2015; 38: 1249-1263.

8. Swaab DF. Prader-Willi syndrome and the hypothalamus. Acta Paediatr Suppl. 1997;423:50-4.

9. DiMario Jr FJ, Burleson JA. Cutaneous blood flow and thermoregulation in Prader-Willi syndrome patients. Pediatr Neurol 2002;26:130-133.

10. Hertz G, Cataletto M, Feinsilver SH, Angulo M. Sleep and breathing patterns in patients with Prader Willi syndrome (PWS): effects of age and gender. Sleep 1993;16:366-371.

11. Stevenson DA, Heinemann J, Angulo $M$, et al. Deaths due to choking in Prader-Willi syndrome. Am J Med Genet A. 2007;143A:484-487.

12. Stevenson DA, Heinemann J, Angulo $M$, et al. Gastric rupture and necrosis in Prader-Willi syndrome. J Pediatr Gastroenterol Nutr. 2007;45:272-274.

13. Hartley SL, Maclean WE Jr, Butler MG, Zarcone J, Thompson T. Maladaptive behaviors and risk factors among the genetic subtypes of Prader-Willi syndrome. Am J Med Genet A. 2005;136:140-145.

14. Hill JO, Kaler M, Spetalnick B, Reed G, Butler MG. Resting metabolic rate in Prader-Willi syndrome. Dysmorphol Clin Genet. 1990;4:27-32.

15. Bittel DC, Butler MG. Prader-Willi syndrome: clinical genetics, cytogenetics and molecular biology. Expert Rev Mol Med. 2005;7:1-20.

16. Butler MG. Prader-Willi syndrome: obesity due to genomic imprinting. Curr Genomics 2011;12:204-15.

17. Kim SJ, Miller JL, Kuipers PJ, et al. Unique and atypical deletions in Prader-Willi syndrome reveal distinct phenotypes. Eur J Hum Genet. 2011;20:283-290.

18. Schwenk WF 2nd. Growth hormone therapy-established uses in short children. Acta Paediatr Suppl. 2006;95:6-8.

19. Butler MG, Manzardo AM, Forster JL. Prader-Willi syndrome: clinical genetics and diagnostic aspects with treatment approaches. Curr Pediatr Rev. 2016;12:136-66.

20. Butler MG. Single gene and syndromic causes of obesity: illustrative examples. Prog Mol Biol Transl Sci. 2016;140:1-45.

21. Butler MG, Lee J, Cox DM, et al. Growth charts for Prader-Willi syndrome during growth hormone treatment. Clin Pediatr 2016;55:957-974.

22. Butler MG, Manzardo AM, Heinemann J, Loker C, Loker J. Causes of death in Prader-Willi syndrome: Prader-Willi Syndrome Association (USA) 40-year mortality survey. Genet Med 2017;19:635-642.

23. Schrander-Stumpel CT, Curfs LM, Sastrowijoto P, Cassidy SB, Schrander $\mathrm{JJ}$, Fryns JP. Prader-Willi syndrome: causes of death in an international series of 27 cases. Am J Med Genet A. 2004;124A:333-338.

24. Van Vliet G, Deal CL, Crock PA, Robitaille Y, Oligny LL. Sudden death in growth hormone-treated children with Prader-Willi syndrome. J Pediatr. 2004;144:129-131.

25. Eiholzer U. Deaths in children with Prader-Willi syndrome. Horm Res. 2005;63:33-39.

26. Nagai T, Obata $\mathrm{K}$, Tonoki $\mathrm{H}$, et al. Cause of sudden, unexpected death of Prader-Willi syndrome patients with or without growth hormone treatment. Am J Med Genet A. 2005;136A:45-48.

27. Tauber M, Diene G, Molinas $C$, Hébert M. Review of 64 cases of death in children with Prader-Willi syndrome (PWS). Am J Med Genet A. 2008;146A:881-887

28. Lionti T, Reid SM, Rowell MM. Prader-Willi syndrome in Victoria: mortality and causes of death. J Paediatr Child Health. 2012;48:506-511.

29. Whittington JE, Holland AJ, Webb T. Ageing in people with Prader-Willi syndrome: mortality in the UK population cohort and morbidity in an older sample of adults. Psychol Med. 2015;45:615-21. 